\title{
Intramuscular versus intravenous oxytocin to prevent postpartum haemorrhage at vaginal delivery: randomised controlled trial
}

\author{
Nita Adnan, ${ }^{1}$ Rebecca Conlan-Trant, ${ }^{1}$ Ciara McCormick, ${ }^{1}$ Fiona Boland, ${ }^{2}$ Deirdre J Murphy ${ }^{1}$
}

\begin{abstract}
${ }^{1}$ Academic Department of
Obstetrics and Gynaecology,

Trinity College, University of

Dublin \& Coombe Women \&

Infants University Hospital,

Dublin 8, Republic of Ireland

${ }^{2}$ HRB Centre for Primary Care

Research, Department of

General Practice, Royal College

of Surgeons in Ireland, Dublin 2,

Republic of Ireland

Correspondence to: DJ Murphy

deirdre.j.murphy@tcd.ie

Additional material is published online only. To view please visit the journal online.

Cite this as: $B M J$ 2018;362:k3546 http://dx.doi.org/10.1136/bmj.k3546

Accepted: 25 July 2018
\end{abstract}

\section{ABSTRACT}

OBJECTIVE

To determine whether intravenous oxytocin is more effective than intramuscular oxytocin at preventing postpartum haemorrhage at vaginal delivery.

DESIGN

Double blind placebo controlled randomised trial.

\section{SETTING}

University affiliated maternity unit in the Republic of Ireland.

\section{PARTICIPANTS}

1075 women aged 18 years or older, at term with a singleton pregnancy who were aiming for a vaginal delivery with an actively managed third stage of labour.

\section{INTERVENTIONS}

Women were allocated to an intravenous bolus of oxytocin (10 IU in $1 \mathrm{~mL}$ given slowly over one minute) and placebo intramuscular injection $(1 \mathrm{~mL} 0.9 \%$ saline) or an intramuscular bolus of oxytocin (10 IU in $1 \mathrm{~mL}$ ) and placebo intravenous injection (1 mL $0.9 \%$ saline given slowly over one minute) at vaginal delivery. Allocation was by a secure web based randomisation service with masking of participants and clinicians to the trial intervention.

\section{MAIN OUTCOME MEASURES}

The primary outcome was postpartum haemorrhage (PPH, measured blood loss $\geq 500 \mathrm{~mL}$ ). Secondary outcomes were severe PPH (measured blood loss $\geq 1000 \mathrm{~mL}$ ), need for blood transfusion, admission to a high dependency unit, and side effects to oxytocin.

RESULTS

Between 4 January 2016 and 13 December 2017, 1075 women were randomised and 1035 (96.3\%) included in the primary and secondary analyses ( 517 in the intravenous oxytocin group and 518 in the intramuscular oxytocin group). The incidence of $\mathrm{PPH}$ was not significantly lower in the intravenous group

\section{WHAT IS ALREADY KNOWN ON THIS TOPIC}

Postpartum haemorrhage (PPH) is one of the leading causes of maternal morbidity and mortality worldwide

Oxytocin is administered routinely to reduce the risk of PPH at vaginal delivery, but the optimal route for administering the drug has not been established

\section{WHAT THIS STUDY ADDS}

Intravenous oxytocin for the third stage of labour results in less frequent severe $\mathrm{PPH}$ (blood loss $\geq 1000 \mathrm{~mL}$ ), need for blood transfusion, and admission to a high dependency unit compared with intramuscular oxytocin

Intravenous oxytocin was not associated with an excess of side effects

(18.8\%, 97/517) compared with intramuscular group (23.2\%, 120/518): adjusted odds ratio 0.75 (95\% confidence interval 0.55 to 1.03 ). The incidence of severe $\mathrm{PPH}$, however, was significantly lower in the intravenous group $(4.6 \%, 24 / 517)$ compared with intramuscular group (8.1\%, 42/518): 0.54 (0.32 to $0.91)$ as was the need for blood transfusion $(1.5 \% \mathrm{v}$ $4.4 \%, 0.31,0.13$ to 0.70$)$ and admission to a high dependency unit (1.7\% v 3.7\%, 0.44, 0.20 to 0.98). The number needed to treat to prevent one case of severe PPH was 29 (95\% confidence interval 16 to 201) and to prevent one case of blood transfusion was 35 (20 to 121). The incidence of side effects to oxytocin was not increased in the intravenous group compared with intramuscular group $(4.1 \% \vee 5.2 \%$, $0.75,0.42$ to 1.35 ).

\section{CONCLUSION}

Intravenous oxytocin for the third stage of labour results in less frequent severe $\mathrm{PPH}$, blood transfusion, and admission to a high dependency unit than intramuscular oxytocin, and without excess side effects.

\section{TRIAL REGISTRATION}

Current Controlled Trials ISRCTN14718882.

\section{Introduction}

Postpartum haemorrhage (PPH) is one of the leading causes of maternal mortality worldwide, accounting for nearly a quarter of maternal deaths. ${ }^{1-3} \mathrm{PPH}$ associated morbidity includes anaemia, maternal exhaustion, difficulty breastfeeding, blood transfusion, emergency surgery, and admission to a high dependency or intensive care unit. ${ }^{2}$ The most common underlying cause of PPH is uterine atony. ${ }^{3} \mathrm{PPH}$ rates have been reported to be increasing over the past 15 years in developed countries, including Australia, Canada, the USA, and Europe. ${ }^{2-6}$

The value of routine uterotonic drugs (oxytocin, ergometrine, misoprostol) in the third stage of labour has been well established, although the optimal agent and route for being administered remain uncertain. Oxytocin is the preferred choice because it has fewer side effects than other uterotonic agents of similar efficacy, and it is the most commonly used agent worldwide. $^{378}$ It can be administered intravenously or intramuscularly. ${ }^{9}$ The intramuscular route has a uterotonic effect within 3-7 minutes, which persists for 30-60 minutes, whereas the response to the intravenous route is almost instantaneous, reaching a plateau concentration at 30 minutes. ${ }^{10}$ The rapid effect of the intravenous route may lower the risk of $\mathrm{PPH}$, but it has been associated with cardiovascular side effects, 
including tachycardia and hypotension. ${ }^{11-16}$ Cochrane systematic reviews report a reduction in the risk of $\mathrm{PPH}$ by $50-60 \%$ with prophylactic oxytocin, effective at any dose ranging from 3 IU to 10 IU, compared with placebo. ${ }^{1117}$ Guidelines from the Royal College of Obstetricians and Gynaecologists (RCOG) ${ }^{7}$ recommend an intramuscular bolus dose of oxytocin $10 \mathrm{IU}$ after delivery, whereas the World Health Organization recommends oxytocin 10 IU intramuscularly or by slow intravenous injection. ${ }^{3}$

In our university affiliated maternity unit with more than 8500 births annually, the traditional practice over several decades had been to administer oxytocin 10 IU intravenously after delivery. In 2010 a new protocol was implemented recommending intramuscular oxytocin in keeping with RCOG guidelines. Concerns were raised by experienced midwives and obstetricians that the incidence of PPH had increased after the introduction of the intramuscular route, which presented an ideal opportunity for the LabOR (Labour Oxytocin Route) randomised controlled trial. We aimed to establish whether intravenous oxytocin $10 \mathrm{IU}$ is more effective than intramuscular oxytocin $10 \mathrm{IU}$ at preventing PPH at vaginal delivery and whether there is a higher incidence of side effects.

\section{Methods}

\section{Study design and participants}

This was a pragmatic double blind, placebo controlled randomised trial carried out in a large Irish maternity unit. Women were eligible for the trial if they were 18

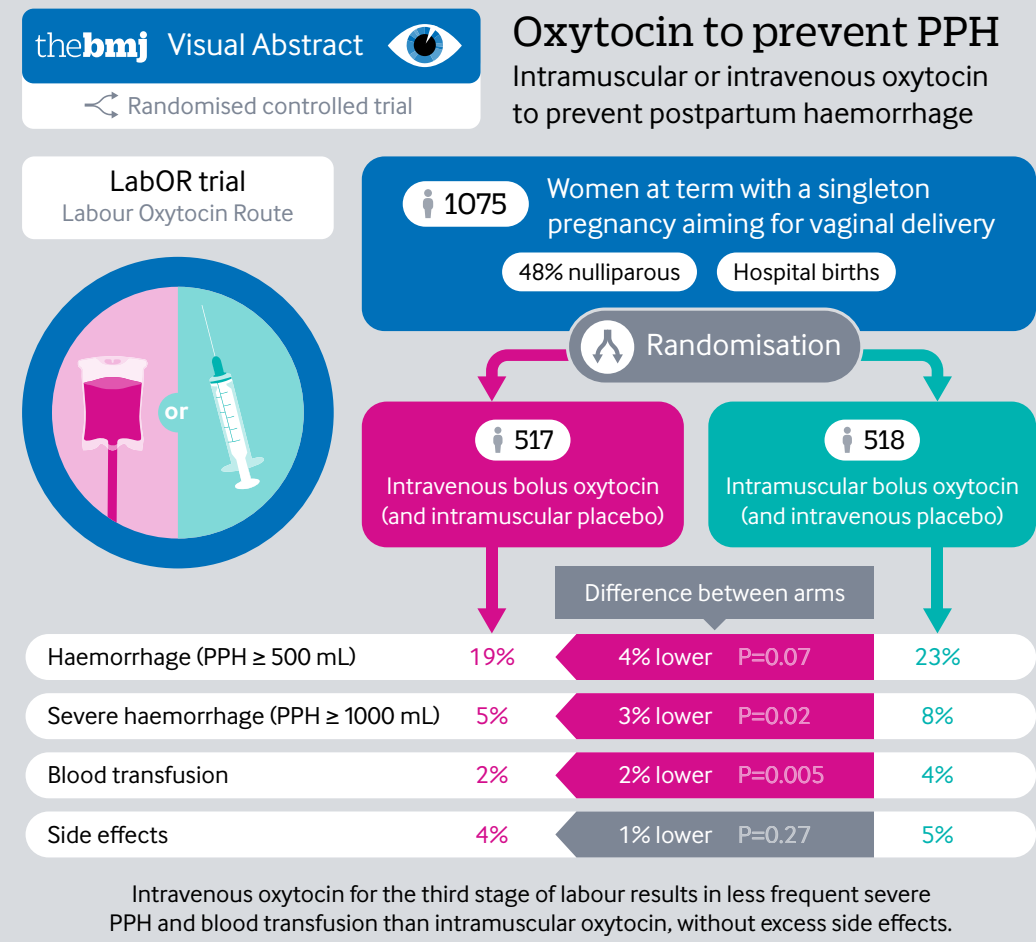

thebmj Read the full article online http://bit.ly/BMJlabor years or older, were at 37 weeks or more gestation, had a singleton pregnancy, and were aiming for a vaginal birth with an actively managed third stage. We excluded women whose caregiver had previously decided to use an additional oxytocin infusion because of an increased risk of $\mathrm{PPH}$, including those with a history of PPH owing to atonic uterus, known fibroids, a history of coagulopathy and receiving anticoagulant treatment, and thrombocytopenia. We excluded women with pre-existing cardiovascular disease and women who did not understand English.

Pregnant women were informed of the study in the outpatient clinics and antenatal classes and provided with an information leaflet that had been approved by the research ethics committees. Recruitment and written consent took place when women attended the assessment unit with symptoms and signs of early labour or when they were admitted for induction. They were eligible to be randomised when the second stage of labour was diagnosed or delivery appeared imminent. Diagnosis was based on the finding of full cervical dilation on vaginal examination, and in parous women, an urge to push.

\section{Randomisation and masking}

The Nottingham Clinical Trials Unit, registered with the UK Clinical Research Collaboration, randomised women to the allocated intervention (allocation ratio 1:1) using a secure automated web based randomisation system. Allocation was stratified by parity (nulliparous or multiparous) and blocked using random permuted blocks of varying size. Women were randomised to receive either an intramuscular bolus of oxytocin (10 IU in $1 \mathrm{~mL}$ ) and a placebo intravenous injection (1 mL $0.9 \%$ saline given slowly over one minute) or an intravenous bolus of oxytocin (10 IU in $1 \mathrm{~mL}$ given slowly over one minute) and a placebo intramuscular injection (1 mL 0.9\% saline).

A research fellow (NA, RC-T, CMcC) randomised women on diagnosis of the second stage of labour and prepared the trial syringes according to the trial allocation. Oxytocin and placebo were drawn up into two separate unlabelled sterile $2 \mathrm{~mL}$ syringes in the labour room and checked by the midwife. This was in keeping with the hospital policy for drawing up drugs and the stipulation of the research ethics committee. The labelling of the syringes-Drug 1-IM or Drug 2-IV injection-then took place outside the labour room according to the randomised allocation. The syringes were returned to the midwife in the labour room. This ensured blinding of everyone except the research fellow, who then played no subsequent role in patient management or outcome assessment.

\section{Procedures}

Women who were confirmed to be in labour or ready to have their labour induced, were brought into a room in the delivery suite. A sample was taken for a full blood count at the time of siting a venous cannula. The first and second stages of labour were managed as usual. To overcome potential variation in the actual practice 
of active management of the third stage of labour, a working definition was specified. Immediately after delivery and preferably within one minute of birth Drug 1-IM was administered intramuscularly into the thigh muscle followed by Drug 2-IV administered intravenously over one minute through a cannula. The baby could be positioned on the mother's chest or abdomen after the birth while the cord was intact and immediate newborn care could be commenced. Owing to the effects of gravity on placental transfusion, babies should not be raised above this level with the cord still intact ${ }^{18}$ At about one to three minutes after the birth the cord was clamped and cut in keeping with the recommendations of WHO and RCOG guidelines. ${ }^{37}$ However, early cord clamping was indicated in emergency situations, such as in the presence of a tight nuchal cord or if immediate resuscitation was required for asphyxia. The placenta was delivered by controlled cord traction once signs of separation were apparent (uterine contraction, show of blood, and apparent cord lengthening) while applying counter traction to the uterus with the other hand. To ensure the membranes do not tear, the placenta should be held in both hands and rotated carefully.

It is widely reported that clinicians underestimate rather than overestimate blood loss and for this study we formally measured blood loss rather than relying on subjective estimates. ${ }^{19}$ We measured blood loss after vaginal deliveries by direct collection of blood and the gravimetric method. After a normal vaginal birth, a fresh delivery sheet was placed under the mother's buttocks as soon as the baby was placed on her chest or abdomen. Once there was no concern of further active bleeding, the blood loss was estimated by weighing all the soiled materials (swabs, pads, disposable sheets) on a scales and subtracting the known weights of these materials. Women remained on the delivery suite for a minimum of one hour after the birth and were monitored throughout that period. They were then transferred to the postnatal ward if there were no apparent complications.

For women who had an operative vaginal delivery, a surgical drape (Barrier Under Buttocks Drape; Mölnlyke Healthcare, Sweden) with a pouch for collecting vaginal blood loss, was placed under the woman's buttocks in preparation for birth and before delivery of the placenta. The bag is transparent, allowing continuous monitoring of blood loss. The bag was left in situ until the birth attendant was no longer concerned about blood loss, such as when a sanitary towel was applied to the vulva and the legs were removed from the lithotomy position. The blood collected in the surgical drape was weighed. Blood soaked swabs and additional drapes were also weighed and the known dry weight of the swabs and drapes subtracted. This volume was added to the measured blood volume from the surgical drape.

After spontaneous vaginal births, the women were monitored on the labour ward for one hour.

The standard practice for recording blood loss and reporting $\mathrm{PPH}$ is based on the midwives' subjective estimate of blood loss from the soaked pads, incontinence sheets, and drapes. We used this record of PPH for the intention to treat analyses for the small number of women whose blood loss was not formally measured.

Maternal vital signs were recorded after delivery and before transfer to the postnatal ward. We used an observation form to record side effects during the first hour after birth. Deviations from the standard procedure were recorded. If the uterus remained atonic despite the trial intervention, the midwife, obstetrician, or anaesthetist could use any additional uterotonic agents according to the hospital PPH protocol. Any major blood loss within 24 hours of delivery was also recorded. A full blood count was performed at day 1 (24 hours) after delivery to assess haemoglobin levels and haematocrit. Clinical follow-up of the mother continued until hospital discharge, and those who were readmitted with a maternal postnatal complication up to six weeks after the birth were flagged up.

\section{Outcomes}

PPH is defined by WHO as a blood loss of $500 \mathrm{~mL}$ or more within 24 hours after birth, and severe PPH is defined as a blood loss of $1000 \mathrm{~mL}$ or more within the same timeframe. ${ }^{3}$ Our primary outcome measure was PPH of $500 \mathrm{~mL}$ or more. We chose a measured blood loss of $500 \mathrm{~mL}$ or more as a clinically relevant outcome and to allow direct comparisons with other studies. Secondary outcomes addressed important measures of clinically relevant $\mathrm{PPH}$, including severe $\mathrm{PPH}$ (measured blood loss $\geq 1000 \mathrm{~mL}$ ), need for blood transfusion, mean total estimated blood loss, and admission to a high dependency unit. Additional secondary outcomes were the complications and consequences of $\mathrm{PPH}$, including the need for additional uterotonic agents or additional surgical procedures, change in haemoglobin levels and haematocrit, severe anaemia (decrease in haemoglobin levels by $\geq 20 \%) 24$ hours after delivery, and length of postnatal stay in hospital. The incidence of side effects after oxytocin treatment was recorded, including nausea, vomiting, hypotension, and tachycardia. We defined hypotension as a fall in blood pressure of more than $30 \%$ below the predelivery blood pressure or use of ephedrine, or both, and tachycardia as a heart rate persistently greater than 100 beats per minute. We recorded breastfeeding initiation within one hour of birth and at the time of discharge and readmissions for maternal postnatal complications up until six weeks post partum.

\section{Statistical analysis}

We estimated from local and national reports that 6-10\% of study participants would have a blood loss of $500 \mathrm{~mL}$ or more. ${ }^{620}$ These estimates were based on observed recordings of $\mathrm{PPH}$ and it was therefore likely that the rate of PPH based on measured blood loss would be higher, consistent with our findings on the Elective caesarean Section Syntocinon Infusion Trial. ${ }^{21}$ With 500 patients in each group for analysis, the study would have $80 \%$ power at the $5 \%$ two 
sided $\alpha$ level to detect differences in the proportion of patients with a measured blood loss of $500 \mathrm{~mL}$ or more of $10 \%$ versus $5 \%$, or $15 \%$ versus $9 \%$, equivalent to odds ratios of 0.50 and 0.55 , respectively. We inflated the final sample size to allow for women who subsequently delivered by caesarean section, and for missing outcome data or deviations from the trial protocol.

Data reporting was according to CONSORT guidelines for randomised controlled trials, and the trial statistician and research team conducted analyses blinded to group status. ${ }^{22}$ The statistical analysis plan was agreed before trial completion as described in the study protocol. ${ }^{23}$ As we aimed to evaluate PPH at vaginal delivery, we excluded women who delivered by caesarean section after randomisation but accounted for them in the CONSORT diagram.

For the first stage of analysis we used descriptive statistics to describe recruited women and to investigate comparability of the trial arms at baseline. The primary analyses involved intention to treat comparisons between the two groups for the primary and secondary outcomes adjusted for operative vaginal delivery and the stratification variable (parity). Secondary analyses investigated the effects of further adjustment for any variables displaying imbalance between the arms at baseline. All analyses used logistic or linear regression models, with results presented as point estimates (odds ratios or difference in means), 95\% confidence intervals, and $\mathrm{P}$ values. We calculated the number needed to treat (NNT) with 95\% confidence intervals for primary and secondary PPH outcomes. The NNT is the reciprocal of the absolute risk difference, or 1 / adjusted risk ratio. ${ }^{24}$

Planned subgroup analyses evaluated the effects of parity and whether the onset of labour was spontaneous or induced. The analyses were replicated for women who completed the trial as per protocol, excluding those who were randomised but did not receive the trial intervention or who did not have blood loss measured (see supplementary tables).

Assessed for eligibility $(n=2510)$

\begin{tabular}{|c|c|}
\hline & $\begin{array}{l}\text { Excluded ( } n=1038) \text { : } \\
\text { Not meeting inclusion criteria }(n=557) \\
\text { Declined to participate }(n=108) \\
\text { Inappropriate to consent }(n=83) \\
\text { Advanced labour or delivering }(n=290) \\
\text { Consented total }(n=1472) \\
\text { Consented not randomised }(n=397) \text { : } \\
\text { Researcher not available }(n=361) \\
\text { Lower segment caesarean section (LSCS) } \\
\text { in first stage }(n=36)\end{array}$ \\
\hline \multicolumn{2}{|c|}{ Randomised $(n=1075)$} \\
\hline$\downarrow$ & $\downarrow$ \\
\hline $\begin{array}{l}\text { Excluded owing to LSCS }(n=20) \\
\text { Eligible intravenous group }(n=517) \text { : } \\
\text { Received allocated intervention }(n=493) \\
\text { Deviation from protocol }(n=24)\end{array}$ & $\begin{array}{l}\text { Excluded owing to LSCS }(n=20) \\
\text { Eligible intravenous group }(n=518) \text { : } \\
\text { Received allocated intervention }(n=507) \\
\text { Deviation from protocol }(n=11)\end{array}$ \\
\hline
\end{tabular}

Fig 1 | Participant flow through the study
The trial steering committee met quarterly. An independent data monitoring committee was established and reviewed recruitment and safety data after 700 women had been recruited. The women provided written informed consent.

\section{Patient and public involvement}

An initial pilot phase was completed, which helped inform the design of the main trial and the recruitment procedures. Recruited women were asked to provide feedback on how they were approached, the timing of recruitment and the consent procedures, blood testing and cannula insertion, and the presence of the researcher in the second stage of labour to prepare the trial drug syringes. Additional insights were provided by the lay members of the institutional research ethics committee, particularly with drafting of the patient information leaflet.

\section{Results}

Between 4 January 2016 and 13 December 2017, 1075 women were randomised to the LabOR trial and 1035 (96.3\%) were included in the primary and secondary analyses on an intention to treat basis ( 517 in the intravenous group and 518 in the intramuscular group). A total of 40 women (3.7\%) were ineligible after randomisation as their babies were delivered by caesarean section. A further 35 women $(3.3 \%$; 24 in the intravenous group and 11 in the intramuscular group) did not receive the allocated intervention (usually because intravenous treatment was specified by the clinician) or have blood loss measured formally and were excluded from the per protocol analyses. A total of 11 women (eight in the intravenous group and three in the intramuscular group) requested the intervention after cord pulsation had ceased. Routinely collected data were available for $100 \%$ of the recruited women. Paired blood tests before and 24 hours after delivery were available for 405/517 (78.3\%) women in the intravenous group and 410/518 (79.1\%) in the intramuscular group, reflecting early postnatal discharge of uncomplicated women. Figure 1 shows the participant flow.

Baseline characteristics were similar between the trial arms (table 1). Almost 30\% of women were 35 years or older, $12 \%$ were obese, and approximately half were nulliparous, similar to the maternity population in Ireland. ${ }^{25}$ Labour was induced in over half the women, and one in three women required augmentation with an oxytocin infusion. Anaemia was diagnosed in $7 \%$ of women during pregnancy, and two thirds reported taking iron supplements, which are routinely recommended in Ireland. The spontaneous vaginal delivery rate was almost $70 \%$, with a slightly higher operative vaginal delivery rate in the intravenous group. Given the strong association between operative vaginal delivery and $\mathrm{PPH}$ we included this factor in the adjusted regression analyses. Episiotomy and perineal tear rates were similar as was the distribution of infant sex and high birthweight babies ( $\geq 4000 \mathrm{~g})$. 


\begin{tabular}{|c|c|c|}
\hline \multirow[b]{2}{*}{ Characteristics } & \multicolumn{2}{|l|}{ No (\%) } \\
\hline & Intravenous oxytocin $(\mathrm{n}=517)$ & Intramuscular oxytocin $(n=518)$ \\
\hline Maternal age $\geq 35$ years & $153(29.6)$ & $145(28.0)$ \\
\hline Obese (BMI $\geq 30.0)$ & $60(11.6)$ & $69(13.3)$ \\
\hline Nulliparous & $253(48.9)$ & $250(48.3)$ \\
\hline \multicolumn{3}{|l|}{ Parity: } \\
\hline $1-3$ & $264(51.1)$ & $268(51.7)$ \\
\hline$\geq 4$ & $0(0.0)$ & $0(0.0)$ \\
\hline Previous caesarean section & $22(4.3)$ & $23(4.4)$ \\
\hline Haemoglobin $<105 \mathrm{~g} /$ L pre-delivery* & $32(6.8)$ & $32(6.8)$ \\
\hline Iron supplements taken & $336(65.0)$ & $344(66.4)$ \\
\hline Induction of labour & $271(52.4)$ & $275(53.1)$ \\
\hline Augmentation with oxytocin & $113(21.8)$ & $109(21.0)$ \\
\hline Epidural analgesia & $359(69.4)$ & $359(69.3)$ \\
\hline Prolonged labour (>12 hours) & $33(6.4)$ & $29(5.6)$ \\
\hline Pyrexia in labourt & $19(3.7)$ & $13(2.5)$ \\
\hline Spontaneous vaginal delivery & $347(67.1)$ & $361(69.7)$ \\
\hline Operative vaginal delivery & $170(32.9)$ & $157(30.4)$ \\
\hline Episiotomy & $176(34.0)$ & $161(31.1)$ \\
\hline Second degree perineal tear & $191(36.9)$ & $179(34.6)$ \\
\hline Third or fourth degree perineal tear & $21(4.1)$ & $24(4.6)$ \\
\hline Male infant & $273(52.8)$ & $267(51.1)$ \\
\hline Birth weight $\geq 4000 \mathrm{~g}$ & 76 (14.7) & 74 (14.3) \\
\hline
\end{tabular}

No maternal deaths occurred, no hysterectomies were carried out, and no woman required transfer out of the hospital for intensive care. The incidence of $\mathrm{PPH}$ was lower in the intravenous group than intramuscular group (18.8\% $v 23.2 \%$, respectively), but the difference did not reach statistical significance (absolute difference 4.4\%, adjusted odds ratio 0.75 , $95 \%$ confidence interval 0.55 to 1.03 ) (table 2). However, the incidence of severe $\mathrm{PPH}$ was significantly lower in the intravenous group compared with intramuscular group: $4.6 \% v 8.1 \%$ (adjusted odds ratio $0.54,95 \%$ confidence interval 0.32 to 0.91 ) as was the need for blood transfusion $(1.5 \% v 4.4 \%, 0.31,0.13$ to 0.70$)$. The number needed to treat to prevent one severe case of PPH was 29 (95\% confidence interval 16 to 201) and to prevent one blood transfusion was 35 (20 to 121). The incidence of side effects to oxytocin was not increased in the intravenous group compared with intramuscular group $(4.1 \% \vee 5.2 \%$, adjusted odds ratio $0.75,95 \%$ confidence interval 0.42 to 1.35 ) (table 3). Side effects were predominantly hypotension and tachycardia, as would be expected for oxytocin, and no serious drug reactions occurred (table 4). Fewer women were admitted to a high dependency unit in the intravenous group compared with intramuscular group $(1.7 \% \vee 3.7 \%$, adjusted odds ratio 0.44 , 95\% confidence interval 0.20 to 0.98 ). The incidence of severe anaemia (decrease in haemoglobin levels by $\geq 20 \%$ ) in each group was consistent with the recorded incidence of PPH. Small non-significant differences were found between the groups in the haematological indices before and after delivery that may reflect the higher incidence of blood transfusion in the intramuscular group. The results for the other secondary outcomes were similar. The associations in the subgroup analyses were stronger for nulliparous women and for women who experienced induced labour (table 5).

\section{Discussion}

In this randomised controlled trial of women who delivered vaginally with active management of the third stage of labour, the incidence of postpartum haemorrhage (PPH, $\geq 500 \mathrm{~mL}$ ) was not significantly reduced in those assigned to intravenous oxytocin compared with intramuscular oxytocin but there was a statistically significant reduction in the incidence of severe PPH $(\geq 1000 \mathrm{~mL})$, need for blood transfusion, and admission to a high dependency unit. The number of side effects were not increased in women who received intravenous oxytocin.

\begin{tabular}{|c|c|c|c|c|c|}
\hline Outcomes & $\begin{array}{l}\text { Intravenous } \\
\text { oxytocin }(n=517)\end{array}$ & $\begin{array}{l}\text { Intramuscular } \\
\text { oxytocin }(n=518)\end{array}$ & $\begin{array}{l}\text { Adjusted odds } \\
\text { ratio* }(95 \% \mathrm{Cl})\end{array}$ & $\begin{array}{l}P \\
\text { value }\end{array}$ & $\begin{array}{l}\text { NNT } \\
(95 \% \mathrm{Cl})\end{array}$ \\
\hline Postpartum haemorrhage ( $\geq 500 \mathrm{~mL})$ & $97(18.8)$ & $120(23.2)$ & 0.75 (0.55 to 1.03$)$ & 0.07 & _ \\
\hline Severe postpartum haemorrhage $(\geq 1000 \mathrm{~mL})$ & $24(4.6)$ & $42(8.1)$ & $0.54(0.32$ to 0.91$)$ & 0.02 & 29 (16 to 201) \\
\hline Mean (SD) blood loss (mL) & $385(326)$ & $445(412)$ & 60 (14 to 106$) \dagger$ & 0.01 & \\
\hline Blood transfusion & $8(1.5)$ & $23(4.4)$ & $0.31(0.13$ to 0.70$)$ & 0.005 & $35(20$ to 121$)$ \\
\hline
\end{tabular}

NNT=number needed to treat.

*Adjusted for parity and operative vaginal delivery.

tMean difference $(95 \% \mathrm{Cl})$. 


\begin{tabular}{|c|c|c|c|c|}
\hline Complications & Intravenous oxytocin $(n=517)$ & Intramuscular oxytocin $(n=518)$ & Adjusted odds ratio* $(95 \% \mathrm{Cl})$ & $P$ value \\
\hline Side effects after oxytocint & $21(4.1)$ & $27(5.2)$ & $0.75(0.42$ to 1.35$)$ & 0.34 \\
\hline Additional uterotonic agent $\ddagger$ & $128(24.8)$ & $140(27.0)$ & $0.87(0.65$ to 1.15$)$ & 0.32 \\
\hline Additional surgical procedure§ & $21(4.1)$ & $29(5.6)$ & $0.69(0.39$ to 1.23$)$ & 0.21 \\
\hline Admission to high dependency unit & $9(1.7)$ & $19(3.7)$ & $0.44(0.20$ to 0.98$)$ & 0.04 \\
\hline Prolonged hospital stay (>3 days) & $46(8.9)$ & $41(7.9)$ & $1.11(0.71$ to 1.73$)$ & 0.66 \\
\hline Breastfeeding initiation & $314(60.7)$ & $308(59.5)$ & $1.06(0.82$ to 1.36$)$ & 0.67 \\
\hline Breastfeeding at discharge & $289(55.9)$ & $280(54.1)$ & $1.08(0.84$ to 1.37$)$ & 0.56 \\
\hline Hospital readmission & $4(0.8)$ & $5(1.0)$ & $0.82(0.21$ to 3.00$)$ & 0.74 \\
\hline Haemoglobin decrease $\geq 20 \% 24$ hours after delivery 9 & $100(19.3)$ & $104(20.1)$ & $0.92(0.66$ to 1.29$)$ & 0.63 \\
\hline Mean (SD) haemoglobin decrease post-delivery (g/L) & $16.9(14.1)$ & $18.0(14.9)$ & $1.2(-0.9 \text { to } 3.2)^{\star \star}$ & 0.26 \\
\hline Mean (SD) decrease in haematocrit post-delivery (\%) & $0.047(0.042)$ & $0.050(0.045)$ & $0.003(-0.003 \text { to } 0.009)^{\star \star}$ & 0.29 \\
\hline \multicolumn{5}{|c|}{$\begin{array}{l}\text { *Adjusted for parity and operative vaginal delivery. } \\
\text { †Nausea, vomiting, hypotension, tachycardia. } \\
\text { ¥0xytocin infusion, ergometrine, misoprostol, carboprost. } \\
\text { §Manual removal of placenta, examination under anaesthesia, Rusch balloon tamponade. } \\
\text { १405 in intravenous group and } 410 \text { in intramuscular group had paired blood tests reflecting early discharges before repeat testing. } \\
\star \star \text { Mean difference }(95 \% \mathrm{Cl}) \text {. }\end{array}$} \\
\hline
\end{tabular}

\section{Strengths and limitations of this study}

We conducted a large pragmatic double blind placebo controlled randomised trial in an academic maternity centre in the Republic of Ireland. The eligibility criteria were broad and together with the high enrolment and randomisation rates ensured external validity and generalisability to the wider population of women in labour. It is a challenge to recruit women to a placebo controlled trial that involves intramuscular and intravenous injections in the third stage of labour, but this was essential to facilitate masking of the interventions given the inherent bias among professionals providing care. The women and health professionals in our centre were well disposed to the research question, which resulted in a high rate of participation. We standardised the procedures for the third stage of labour, taking account of the recent recommendation for delayed cord clamping. Blood loss was measured rather than visually estimated, which is an important advance on previous research on $\mathrm{PPH}$. To validate blood loss, we checked full blood counts before and 24 hours after delivery. The single centre design ensured that measurement of blood loss and third stage procedures were carried out consistently, which strengthens the internal validity of the study.

Some limitations of the study warrant consideration. We would have preferred to complete a multicentre study as we have done previously when investigating oxytocin use at caesarean section. ${ }^{21}$ However, potential collaborating centres in Ireland had long established practices for the third stage of labour

\begin{tabular}{|c|c|c|}
\hline \multirow[b]{2}{*}{ Side effects } & \multicolumn{2}{|l|}{ No (\%) } \\
\hline & $\begin{array}{l}\text { Intravenous } \\
\text { oxytocin }(n=517)\end{array}$ & $\begin{array}{l}\text { Intramuscular } \\
\text { oxytocin }(n=518)\end{array}$ \\
\hline Nausea & $1(0.2)$ & $1(0.2)$ \\
\hline Vomiting & $0(0.0)$ & $0(0.0)$ \\
\hline Headache & $3(0.6)$ & $4(0.8)$ \\
\hline Shivering & $2(0.4)$ & $5(1.0)$ \\
\hline Tachycardia & $10(1.9)$ & $14(2.7)$ \\
\hline Hypotension & $12(2.3)$ & $15(2.9)$ \\
\hline
\end{tabular}

using intramuscular oxytocin or syntometrine and were reluctant to randomise women to intravenous oxytocin. We debated the choice of primary outcome and made a pragmatic decision to use $\mathrm{PPH}$ of $500 \mathrm{~mL}$ or more, although blood loss in the range 500-1000 $\mathrm{mL}$ is well tolerated by most women in a developed world setting. Severe PPH $(\geq 1000 \mathrm{~mL})$ is the more relevant clinical outcome as it is a leading cause of maternal death worldwide. We did not anticipate the high number of severe $\mathrm{PPH}$ events, which if known would have influenced the power and sample size calculations. However, although the clinically significant results are based on secondary outcomes and need to be interpreted accordingly, the results for severe PPH, measured blood loss, need for blood transfusion, and admission to a high dependency unit are all consistent, suggesting that this is likely to be a true effect.

\section{Comparison with other studies}

Several Cochrane systematic reviews have addressed the use of uterotonic agents to prevent $\mathrm{PPH}$ at vaginal delivery. 9 111726 The most recent review, updated in 2015, included seven studies comparing active management of the third stage of labour with physiological management using a range of uterotonic agents, including ergometrine, syntometrine, intramuscular oxytocin, and intravenous oxytocin. ${ }^{26}$ Active management was associated with a reduced incidence of PPH irrespective of the agent used, but none of the studies were graded as high quality. A review in 2013 of 20 studies comparing prophylactic oxytocin with placebo or alternative agents reported that prophylactic oxytocin at any dose decreased PPH greater than $500 \mathrm{~mL} .{ }^{11}$ The authors recommended the intravenous route, where the evidence was strongest, with the intramuscular route as an alternative, and concluded that further high quality evidence was required. A Cochrane review published in 2012 that specifically compared intramuscular oxytocin with intravenous oxytocin for the prevention of $\mathrm{PPH}$ at vaginal delivery identified no randomised controlled trials (published or unpublished). ${ }^{9}$ 


\begin{tabular}{|c|c|c|c|c|}
\hline \multirow[b]{2}{*}{ Variables } & \multicolumn{2}{|l|}{ No (\%) } & \multirow[b]{2}{*}{$\begin{array}{l}\text { Adjusted odds } \\
\text { ratio* }(95 \% \mathrm{CI})\end{array}$} & \multirow[b]{2}{*}{$\begin{array}{l}P \\
\text { value }\end{array}$} \\
\hline & $\begin{array}{l}\text { Intravenous } \\
\text { oxytocin }(n=253)\end{array}$ & $\begin{array}{l}\text { Intramuscular } \\
\text { oxytocin }(n=250)\end{array}$ & & \\
\hline \multicolumn{5}{|l|}{ Nulliparous: } \\
\hline $\mathrm{PPH} \geq 500 \mathrm{~mL}$ & $61(24.1)$ & $80(32.0)$ & $0.69(0.45$ to 1.02$)$ & 0.06 \\
\hline Severe $\mathrm{PPH} \geq 1000 \mathrm{~mL}$ & $16(6.3)$ & $33(13.2)$ & $0.45(0.24$ to 0.84$)$ & 0.01 \\
\hline Multiparous: & $n=264$ & $\mathrm{n}=268$ & & \\
\hline $\mathrm{PPH} \geq 500 \mathrm{~mL}$ & $36(13.6)$ & $40(14.9)$ & $0.90(0.55$ to 1.46$)$ & 0.67 \\
\hline Severe $\mathrm{PPH} \geq 1000 \mathrm{~mL}$ & $8(3.0)$ & $9(3.4)$ & $0.92(0.35$ to 2.42$)$ & 0.86 \\
\hline Induction of labour: & $n=271$ & $n=275$ & & \\
\hline $\mathrm{PPH} \geq 500 \mathrm{~mL}$ & $50(18.5)$ & $64(23.3)$ & 0.75 (0.49 to 1.13$)$ & 0.71 \\
\hline Severe $\mathrm{PPH} \geq 1000 \mathrm{~mL}$ & $10(3.7)$ & $28(10.2)$ & $0.34(0.16$ to 0.72$)$ & 0.005 \\
\hline Spontaneous labour: & $n=246$ & $n=243$ & & \\
\hline $\mathrm{PPH} \geq 500 \mathrm{~mL}$ & $47(19.1)$ & $56(23.0)$ & $0.82(0.53$ to 1.26$)$ & 0.36 \\
\hline Severe $\mathrm{PPH} \geq 1000 \mathrm{~mL}$ & $14(5.7)$ & $14(5.8)$ & $1.01(0.47$ to 2.16$)$ & 0.99 \\
\hline
\end{tabular}

Two relevant randomised controlled trials have been reported since the latest Cochrane review. One study of 256 women compared an intravenous oxytocin infusion $10 \mathrm{IU}$ in $1 \mathrm{~L}$ normal saline infused at a rate of $1 \mathrm{~mL} / \mathrm{min}$ with intramuscular oxytocin 10 IU. Blinding of the clinicians, participants, and researchers was not mentioned and no difference was found in measured blood loss or $\mathrm{PPH} .{ }^{27}$ The other study compared oxytocin $10 \mathrm{IU}$ intravenous at $1 \mathrm{~mL} /$ min with oxytocin $10 \mathrm{IU}$ intramuscularly. ${ }^{28}$ Birth attendants and participants were not blinded but the research team who performed the measurement of blood loss were blinded. The design was factorial, with 150 participants in each group, and haematological indices did differ but not measured blood loss. The lack of effect in these two studies is not surprising given that the approach was to administer a low dose intravenous infusion over several hours, which provides a sustained effect but not the immediate uterotonic effect of an intravenous bolus. The optimal approach to use of oxytocin in the third stage of labour is therefore a poorly evaluated component of maternity care, despite its widespread use.

\section{Conclusions and policy implications}

$\mathrm{PPH}$ is common, even in women apparently at low risk. Our findings support the use of intravenous oxytocin rather than intramuscular oxytocin in terms of preventing severe $\mathrm{PPH}$ and the need for blood transfusion, with the potential to prevent mortality if implemented globally in developing world settings.

Implementation of the trial findings in some settings will require a change in established practice. A survey of more than 4000 health professionals in the United Kingdom reported that intramuscular syntometrine (oxytocin-ergometrine combined) was the uterotonic agent used routinely by $86 \%$ of midwives and $79 \%$ of obstetricians, although most respondents thought more evidence from randomised trials was needed. ${ }^{29}$ This finding was surprising given that guidelines from the National Institute for Health and Care Excellence, World Health Organization, and Royal College of
Obstetricians and Gynaecologists clearly recommend oxytocin as the prophylactic agent of choice for the third stage of labour. ${ }^{378}$ It may be that where syntometrine has been the long established uterotonic agent for third stage prophylaxis, intramuscular syntocinon is perceived as less effective, similar to the perception in our unit that intramuscular oxytocin was inferior to intravenous oxytocin. However, side effects of syntometrine include nausea, vomiting, and headache and it is contraindicated in women with hypertension. In the context of this new evidence, health professionals are more likely to consider using intravenous oxytocin as an alternative to intramuscular syntometrine. In a global setting oxytocin is the preferred agent, and implementation of the trial findings through WHO initiatives will be far easier to achieve. ${ }^{3-5}$

The risk of adverse cardiovascular events associated with intravenous oxytocin also needs to be addressed. ${ }^{1216}$ Oxytocin has a direct relaxing effect on vascular smooth muscle, which can lead to a transient decrease in systemic vascular resistance resulting in hypotension and tachycardia. These haemodynamic responses have been mainly associated with the intravenous route when given by rapid bolus injection in women under anaesthesia for caesarean delivery. ${ }^{16}$ In the Confidential Enquiry into Maternal Death Report of 1997-99, the death of two mothers with cardiovascular instability was related to cardiac arrest after intravenous injection of 10 IU oxytocin. ${ }^{12}$ The resulting concerns led to a call for caution in using intravenous oxytocin in women with unstable cardiovascular conditions such as hypovolaemia, shock, or cardiac disease. A haemodynamic study completed in advance of the Elective caesarean Section Syntocinon Infusion Trial showed that circulatory disturbances in otherwise healthy women occurred secondary to regional anaesthesia and not in response to intravenous bolus oxytocin. ${ }^{30}$ The current study provides important evidence on the safety of intravenous oxytocin $10 \mathrm{IU}$ administered as a slow bolus injection over one minute after exclusion of women with a history of cardiovascular disease. The overall incidence of side effects was low and in keeping with the safety data on intravenous oxytocin in the context of caesarean births. ${ }^{21}$

\section{Conclusion}

Intravenous oxytocin for the third stage of labour results in less frequent severe $\mathrm{PPH}$, need for blood transfusion, and admission to a high dependency unit than intramuscular oxytocin, and without excess side effects. These findings should inform decision making when advising women on management options for the third stage of labour.

We thank the women who participated in the study; the midwives and medical staff who provided support; Ann Fergus for her midwifery expertise; Clare Dunney and Farah Nazir for their assistance with the early stages of recruitment; the data monitoring and ethics committee, Alan Montgomery (chair) and Declan Devane, and the independent chair of the trial steering committee, Caoimhe Lynch for their support; and Tom Fahey, the reviewers, and the editorial committee for helpful feedback on the manuscript. 
Contributors: DJM had the original idea for the study and with NA and FB designed the protocol. NA, RC-T, and CMcC recruited the women and allocated the interventions. DJM and FB carried out the analysis. DJM and NA drafted the manuscript, which was revised by all authors. DJM acts as guarantor. The corresponding author attests that all listed authors meet authorship criteria and that no others meeting the criteria have been omitted.

Funding: This study was funded by Trinity College, University of Dublin and Coombe Women and Infants University Hospital. Coombe Women and Infants University Hospital sponsored the study.

Competing interests: All authors have completed the ICMJE uniform disclosure form at http://www.icmje.org/coi_disclosure.pdf (available on request from the corresponding author) and declare: no support from any organisation for the submitted work; no financial relationships with any organisations that might have an interest in the submitted work in the previous three years; no other relationships or activities that could appear to have influenced the submitted work.

Ethical approval: The protocol (01/2012) was approved by the National Maternity Hospital research ethics committee and the Coombe Women and Infants University Hospital research ethics committee (26-2015)

Data sharing: Requests for access to data from the LabOR trial should be addressed to the corresponding author.

Transparency: The lead author (DJM) affirms that the manuscript is a honest, accurate, and transparent account of the study bring reported; that no important aspects of the study have been omitted; and that any discrepancies from the study as planned (and, if relevant, registered) have been explained.

This is an Open Access article distributed in accordance with the Creative Commons Attribution Non Commercial (CC BY-NC 4.0) license, which permits others to distribute, remix, adapt, build upon this work non-commercially, and license their derivative works on different terms, provided the original work is properly cited and the use is noncommercial. See: http://creativecommons.org/licenses/by-nc/4.0/.

1 Khan KS, Wojdyla D, Say L, Gülmezoglu AM, Van Look PFA. WHO analysis of causes of maternal death: a systematic review. Lancet 2006;367:1066-74. doi:10.1016/S0140-6736(06)68397-9

2 Hogan MC, Foreman KJ, Naghavi M, et al. Maternal mortality for 181 countries, 1980-2008: a systematic analysis of progress towards Millennium Development Goal 5. Lancet 2010;375:1609-23. doi:10.1016/S0140-6736(10)60518-1

3 Department of Reproductive Health and Research WHO. WHO Recommendations for the prevention and treatment of postpartum haemorrhage. World Health Organization, 2012.

4 Al-Zirqi I, Vangen S, Forsen L, Stray-Pedersen B. Prevalence and risk factors of severe obstetric haemorrhage. BJOG 2008;115:1265-72. doi:10.1111/j.1471-0528.2008.01859.x

5 Knight M, Callaghan WM, Berg C, et al. Trends in postpartum hemorrhage in high resource countries: a review and recommendations from the International Postpartum Hemorrhage Collaborative Group. BMC Pregnancy Childbirth 2009;9:55 doi:10.1186/1471-2393-9-55

6 Lutomski JE, Byrne BM, Devane D, Greene RA. Increasing trends in atonic postpartum haemorrhage in Ireland: an 11-year population-based cohort study. BJOG 2012;119:306-14. doi:10.1111/j.1471-0528.2011.03198.x

7 Prevention and management of postpartum haemorrhage. Green top Guideline. RCOG 2009

8 National Institute for Health and Clinical Excellence. Normal labour: third stage. Intrapartum care: care of healthy women and their babies during childbirth, guideline 55. National Institute for Health and Clinical Excellence, 2007

9 Oladapo OT, Okusanya BO, Abalos E. Intramuscular versus intravenous prophylactic oxytocin for the third stage of labour. Cochrane Database Syst Rev 2012;(2):CD009332.

10 Rall TW. Oxytocin, prostaglandins, ergot alkaloids and other drugs; tocolytic agents. In: Gillman AG, Rall TW, Nies AS, Taylor I, eds. The pharmacological basis of therapeutics. Pergamon Press Inc, 1990 933-53.
11 Westhoff G, Cotter AM, Tolosa JE. Prophylactic oxytocin for the third stage of labour to prevent postpartum haemorrhage. Cochrane Database Syst Rev 2013;(10):CD001808

12 Lewis G, Drife J, eds. Why Mothers Die 1997-1999. The fifth report of the Confidential Enquiries into Maternal Deaths in the United Kingdom. RCOG.

13 Soriano D, Dulitzki M, Schiff E, Barkai G, Mashiach S, Seidman DS. A prospective cohort study of oxytocin plus ergometrine compared with oxytocin alone for prevention of postpartum haemorrhage. Br J Obstet Gynaecol 1996;103:1068-73. doi:10.1111/j.1471-0528.1996.tb09584.x

14 Choy CMY, Lau WC, Tam WH, Yuen PM. A randomised controlled trial of intramuscular syntometrine and intravenous oxytocin in the management of the third stage of labour. BJOG 2002;109:173-7. doi:10.1111/j.1471-0528.2002.01204.X

15 Davies GAL, Tessier JL, Woodman MC, Lipson A, Hahn PM. Maternal hemodynamics after oxytocin bolus compared with infusion in the third stage of labor: a randomized controlled trial. Obstet Gynecol 2005:105:294-9. doi:10.1097/01. AOG.0000148264.20909.bb

16 Thomas IS, Koh SH, Cooper GM. Haemodynamic effects of oxytocin given as i.v. bolus or infusion on women undergoing Caesarean section. BrJ Anaesth 2007;98:116-9. doi:10.1093/bja/ael302

17 Begley CM, Gyte GM, Murphy DJ, Devane D, McDonald SJ, McGuire W. Active versus expectant management for women in the third stage of labour. Cochrane Database Syst Rev 2010;(7):CD007412.

18 Clamping of the Umbilical Cord and Placental Transfusion RCOG, Scientific Impact Paper No. 14, February 2015.

19 Rath WH. Postpartum hemorrhage--update on problems of definitions and diagnosis. Acta Obstet Gynecol Scand 2011;90:421-8. doi:10.1111/j.1600-0412.2011.01107.x

20 Annual Report 2014. Coombe Women \& Infants University Hospital, Republic of Ireland

21 Sheehan SR, Montgomery AA, Carey M, et al, ECSSIT Study Group. Oxytocin bolus versus oxytocin bolus and infusion for control of blood loss at elective caesarean section: double blind, placebo controlled, randomised trial. BMJ 2011;343:d4661. doi:10.1136/bmj.d4661

22 Schulz KF, Altman DG, Moher D, CONSORT Group. CONSORT 2010 statement: updated guidelines for reporting paralle group randomized trials. Ann Intern Med 2010;152:726-32. doi:10.7326/0003-4819-152-11-201006010-00232

23 Adnan N, Boland F, Murphy DJ. Intramuscular oxytocin versus intravenous oxytocin to prevent postpartum haemorrhage at vaginal delivery (LabOR trial): study protocol for a randomised controlled trial. Trials 2017:18:541. doi:10.1186/s13063-017-2269-9

24 Altman DG. Confidence intervals for the number needed to treat. BMJ 1998;317:1309-12. doi:10.1136/bmj.317.7168.1309

25 Central Statistics Office. Vital Statistics Annual Report 2014 (http://www.cso.ie/en/releasesandpublications/ep/p-vsar/ vsar2014/births2014/)

26 Begley CM, Gyte GM, Devane D, McGuire W, Weeks A. Active versus expectant management for women in the third stage of labour. Cochrane Database Syst Rev 2015;(3):CD007412.

27 Oguz Orhan E, Dilbaz B, Aksakal SE, Altınbas S, Erkaya S. Prospective randomized trial of oxytocin administration for active management of the third stage of labor. Int J Gynaecol Obstet 2014:127:175-9. doi:10.1016/i.ijgo.2014.05.022

28 Dagdeviren H, Cengiz H, Heydarova U, et al. Intramuscular versus intravenous oxytocin for postpartum haemorrhage after vaginal delivery. Arch Gynecol Obstet 2016;294:911-6. doi:10.1007/s00404-016-4060-7

29 Farrar D, Tuffnell D, Airey R, Duley L. Care during the third stage of labour: a postal survey of UK midwives and obstetricians. BMC Pregnancy Childbirth 2010;10:23. doi:10.1186/1471-2393-10-23

30 McLeod G, Munishankar B, MacGregor H, Murphy DJ. Maternal haemodynamics at elective caesarean section: a randomised comparison of oxytocin 5-unit bolus and placebo infusion with oxytocin 5-unit bolus and 30-unit infusion. Int / Obstet Anesth 2010;19:155-60. doi:10.1016/j.ijoa.2009.08.005

Supplementary information: additional tables, 1-4 